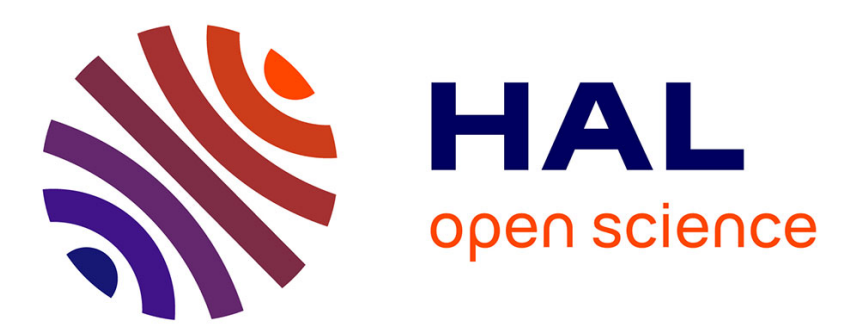

\title{
Model Identification and Validation for translational movements of an Octorotor UAV
}

Majd Saied, Daniel Alshamaa, Hassan Shraim, Clovis Francis, Benjamin

Lussier, Isabelle Fantoni

\section{- To cite this version:}

Majd Saied, Daniel Alshamaa, Hassan Shraim, Clovis Francis, Benjamin Lussier, et al.. Model Identification and Validation for translational movements of an Octorotor UAV. IEEE Workshop on Research, Education and Development of Unmanned Aerial Systems (RED UAS 2015), Nov 2015, Cancún, Mexico. pp.102-108. hal-01263761

\section{HAL Id: hal-01263761 \\ https://hal.science/hal-01263761}

Submitted on 28 Jan 2016

HAL is a multi-disciplinary open access archive for the deposit and dissemination of scientific research documents, whether they are published or not. The documents may come from teaching and research institutions in France or abroad, or from public or private research centers.
L'archive ouverte pluridisciplinaire HAL, est destinée au dépôt et à la diffusion de documents scientifiques de niveau recherche, publiés ou non, émanant des établissements d'enseignement et de recherche français ou étrangers, des laboratoires publics ou privés. 


\title{
Model Identification and Validation for translational movements of an Octorotor UAV
}

\author{
Majd Saied ${ }^{1,2}$, Daniel ALShamaa ${ }^{1}$, Hassan Shraim ${ }^{1}$, Clovis Francis $^{1}$, Benjamin Lussier ${ }^{2}$ and Isabelle Fantoni ${ }^{2}$
}

\begin{abstract}
This paper presents preliminary results on a complete translational system modeling and identification of a coaxial counter-rotating X8 octorotor. The objective is to assess the applicability of the widely-used quadrotor model to the coaxial eight-rotor aircraft. A real and representative model of the UAV is necessary to develop simulation tools for validation and to predict the system behavior. A series of tests were conducted with the $\mathrm{X8}$ in flight when performing movements in the $x, y$ and $z$ directions. The model is simulated and validated offline in a MATLAB environment using real data.
\end{abstract}

\section{INTRODUCTION}

Multirotor unmanned aerial vehicles (UAV) arouse a lot of interest in recent years. Their operation is intended to support human beings activities in numerous applications such as: railway monitoring, supervision of electrical power lines, development of the precision agriculture, package delivery, etc. Different designs exist with a number of rotors greater or equal to four, uniformly distributed in star-shaped or coaxial configurations (quadrotors [1], hexarotors [2], octorotors [3]). Quadrotors are the most popular between the existing multirotors due to their mechanical simplicity and low power requirements. However, octorotors present advantages over quadrotors in terms of improved stability, higher payload capacity and the ability to recover from failures during flight.

These multirotors are inherently unstable systems and hence require precise control in order to operate in a safe manner. Control methodologies range amongst others from proportional-integral-derivative to feedback linearization [6] and backstepping [7], for the control of the orientation, altitude and position. However, implementing these controllers supposes the existence of an accurate system model, taking into consideration nonlinearities and aerodynamic effects.

Extensive work has been done in the literature to model the dynamics of the quadrotor vehicles [1] with various levels of complexity. In [10] and [11], an experimental identification of the quadrotor model parameters is presented. In [12], an open-loop identification of a micro quadrotor LTI model from closed-loop data is developed, followed by

\footnotetext{
1 Université Libanaise, Faculté de Génie, Centre de Recherche Scientifique en Ingénierie (CRSI), Liban, Email: \{cfrancis, hassan.shraim\}@ul.edu.lb,

${ }^{2}$ Sorbonne Universités, Université de Technologie de Compiègne, CNRS, UMR 7253 Heudiasyc, 60200 Compiègne, France, Email: \{majd.saied, benjamin.lussier, isabelle.fantoni\}ehds.utc.fr
}

the application of a linear model-based controller. The last few years witnessed also many developments in the area of dynamics modeling of $n$-rotors helicopters [4], [5]. In most of the applications the modeling was kept relatively simple.

In this paper, we will focus on the translational model validation and identification of a coaxial counter-rotating octorotor UAV. The dynamics equations are deduced from that of a quadrotor [1], using Newton-Euler formalism. The parameters of the translational model are identified using the mean square error minimization criterion. Several tests were conducted on the X8 octorotor in real flights when moving in a single direction along one of the $x, y$ or $z$ axes, or when following a complex trajectory including displacements along the three axes. The real control inputs generated from these experimentations are used as inputs to a simulated model implemented in Matlab. The simulated translational dynamics are then compared to the real measurements obtained during the experimentations from an Optitrack motion capture system (for $\mathrm{x}$ and $\mathrm{y}$ ) and an ultrasonic sensor (for $\mathrm{z}$ ).

This paper is organized as follows: Section II presents the dynamics of the coaxial octorotor and the controller used in closed loop to stabilize the body angles, the altitude and the position in real flights. Section III is dedicated to the model parameters estimation where the experimental platform, the methodology, the experimental data sets and the results are detailed. A discussion on the usability of the obtained results for fault tolerant control and fault diagnosis is presented in section IV, and the paper concludes with perspectives in section $\mathrm{V}$.

\section{DYNAMICS MODELING OF OCTOROTOR}

An octorotor is a highly nonlinear, multivariable, strongly coupled, underactuated, and basically unstable system. It is a 6-DOFs rigid body consisting of eight motors with attached propellers. These motors are fixed at the ends of a number of arms following a symmetric frame. Each motor can be controlled individually, thus modifying the attitude and the altitude of the vehicle and allowing the octorotor to move from one point to another. Different configurations of octorotors exist. According to the arrangement and distribution of rotors, the most widely widespread layouts are coaxial octorotors and star-shaped octorotors. In coaxial octorotors, the actuators are aligned vertically but stacked in pairs so as to resemble a quadrotor, while in star-shaped octorotors 


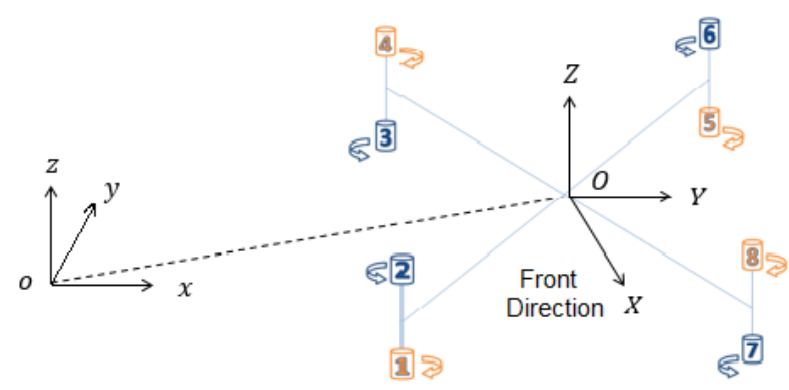

Fig. 1: The coaxial octorotor and the reference frames

the actuators are aligned vertically and are equally spaced around the vehicle. According to the authors in [5], a coaxial octorotor presents advantages over a star-shaped one in terms of endurance and size. This configuration is adopted in this work, and will be controlled using an adapted quadrotor model.

Several methods have been proposed in the literature for the dynamic modeling of the quadrotor vehicle. In [9] and [13], the quadrotor is considered as a rigid body and its dynamics are described using the Euler-Lagrange formalism with small angles approximation. In [14], the model is derived using the Newton-Euler approach. The resultant model from both methods is the same, but written with different notations. In [1], a detailed model of the quadrotor is derived including all rigid body dynamics, aerodynamic forces and gyroscopic effects.

In all the approaches cited above, the models developed assume the following hypotheses :

- The structure of the vehicle is rigid and symmetrical;

- The motor dynamics are ignored;

- The center of gravity and the body fixed frame origin coincide;

- The inertia matrix off-diagonal terms are zero;

- The thrust and the drag are proportional to the square of the rotors speed.

We define two frames that we use to study the system's motion: a body-fixed reference frame $\left\{R_{B}\right\}(O, X, Y, Z)$ originating at the center of mass of the vehicle, and an inertial frame $\left\{R_{I}\right\}(o, x, y, z)$ fixed to the earth. The rotation matrix $R_{I, B}$ defines the attitude of $R_{B}$ with respect to $R_{I}$ :

$R_{I, B}=\left[\begin{array}{ccr}C_{\theta} C_{\psi} & C_{\psi} S_{\theta} S_{\phi}-C_{\phi} S_{\psi} & C_{\phi} C_{\psi} S_{\theta}+S_{\phi} S_{\psi} \\ C_{\theta} S_{\psi} & S_{\theta} S_{\phi} S_{\psi}+C_{\phi} C_{\psi} & C_{\phi} S_{\theta} S_{\psi}-C_{\psi} S_{\phi} \\ -S_{\theta} & C_{\theta} S_{\phi} & C_{\theta} C_{\phi}\end{array}\right]$

where $\phi, \theta$ and $\psi$ are the Euler angles, and $S_{\phi, \theta, \psi}$ and $C_{\phi, \theta, \psi}$ are the sine and the cosine of the corresponding angle.

\section{A. Newton-Euler Formalism}

The dynamics of a rigid body under external forces applied to the center of mass, expressed in the body fixed frame are written in Newton-Euler formalism as follows [1]:

$$
\left[\begin{array}{cc}
m I & 0 \\
0 & I
\end{array}\right]\left[\begin{array}{l}
\dot{V} \\
\dot{\omega}
\end{array}\right]+\left[\begin{array}{c}
\omega \times m V \\
\omega \times I \omega
\end{array}\right]=\left[\begin{array}{c}
F \\
\tau
\end{array}\right]
$$

where $I \in R^{3 \times 3}$ is the inertia matrix, $m$ the mass of the vehicle, $V$ the body linear speed vector and $\omega$ the body angular speed.

To make the model of the octorotor more realistic, hub forces, rolling, pitching and yawing moments and variable aerodynamical coefficients should be considered. The forces acting on the octorotor in the inertial frame $R_{I}$ are listed below:

- Along the $z$-axis:

- actuators action: $C_{\psi} C_{\phi} * u_{f}$

- weight: $m g$

- Along the $x$-axis

- actuators action: $\left(S_{\psi} S_{\phi}+C_{\psi} S_{\theta} C_{\phi}\right) * u_{f}$

- hub force: $-\sum_{i=1}^{8} H_{x_{i}}$

- friction: $\frac{1}{2} C_{x} A_{c} \rho \dot{x}|\dot{x}|$

- Along the $y$-axis

- actuators action: $\left(-C_{\psi} S_{\phi}+S_{\psi} S_{\theta} C_{\phi}\right) * u_{f}$

- hub force: $-\sum_{i=1}^{8} H_{y_{i}}$

- friction: $\frac{1}{2} C_{y} A_{c} \rho \dot{y}|\dot{y}|$

where $H_{x}$ and $H_{y}$ are the hub forces in the $x$ and $y$ directions respectively, $C_{x}, C_{y}$ are friction coefficients, $A_{c}$ is the fuselage area and $\rho$ is the air density.

The moments around the three axes resulting from the generated forces are also listed below:

- Rolling moments

- body gyro effect: $\dot{\theta} \dot{\psi}\left(I_{y y}-I_{z z}\right)$

- propeller gyro effect: $J_{r} \dot{\theta} \Omega_{r}$

- roll actuators action: $\tau_{\phi}$

- Pitching moments

- body gyro effect: $\dot{\phi} \dot{\psi}\left(I_{z z}-I_{x x}\right)$

- propeller gyro effect: $J_{r} \dot{\phi} \Omega_{r}$

- pitch actuators action: $\tau_{\theta}$

- Yawing moments

- body gyro effect: $\dot{\theta} \dot{\phi}\left(I_{x x}-I_{y y}\right)$

- inertial counter-torque: $J_{r} \Omega_{r}$

- counter-torque unbalance: $\tau_{\psi}$

where $J_{r}$ is the rotor inertia and $\Omega_{r}$ is the overall residual propeller speed from the unbalanced rotor rotation, expressed in function of the motors speeds $\omega_{i}$ as follows:

$$
\Omega_{r}=\omega_{2}+\omega_{3}+\omega_{6}+\omega_{7}-\omega_{1}-\omega_{4}-\omega_{5}-\omega_{8}
$$

Note that hub forces also create moments around the three axes, but they are not detailed here.

The total thrust $u_{f}$ defined in $R_{B}$ and the torques $\tau_{\phi}, \tau_{\theta}$ and $\tau_{\psi}$ are defined as:

$$
\begin{aligned}
& u_{f}=F_{12}+F_{34}+F_{56}+F_{78} \\
& \tau_{\phi}=\left(F_{78}+F_{56}-F_{34}-F_{12}\right) * l * \sqrt{2} / 2 \\
& \tau_{\theta}=\left(F_{34}+F_{56}-F_{78}-F_{12}\right) * l * \sqrt{2} / 2 \\
& \tau_{\psi}=\left(\tau_{2}+\tau_{3}+\tau_{6}+\tau_{7}\right)-\left(\tau_{1}+\tau_{4}+\tau_{5}+\tau_{8}\right)
\end{aligned}
$$


The thrust force and the torque produced by each propeller is proportional to the square of the angular velocity:

$$
\begin{aligned}
& F_{i}=K_{f} \omega_{i}^{2} \\
& \tau_{i}=K_{t} \omega_{i}^{2}
\end{aligned}
$$

$l$ is the arm length, $K_{f}$ and $K_{t}$ are the thrust and drag coefficients.

The thrust produced by each pair of coaxial rotors $i$ and $j$ is given by [17]:

$$
\begin{gathered}
F_{i j}=\alpha_{i j} *\left(F_{i}+F_{j}\right) *\left(1+\frac{S_{s}}{S_{p r o p}}\right) \\
F_{i}=K_{f} * \omega_{i}^{2}
\end{gathered}
$$

$\alpha_{i j}$ is the coefficient of loss of aerodynamic efficiency due to the aerodynamic interference between the upper and lower rotors of each pair of coaxial rotors. $S=\left(1+\frac{S_{s}}{S_{\text {prop }}}\right)$ represents the shape factor of the propellers, with $S_{s}$ denoting the propeller's surface and $S_{\text {prop }}$ the surface of the circle that the propeller would make when rotating.

The equations of motion of the octorotor are derived from (2) and all the forces and moments previously given:

$$
\left\{\begin{array}{l}
I_{x x} \ddot{\phi}=\dot{\theta} \dot{\psi}\left(I_{y y}-I_{z z}\right)+J_{r} \dot{\theta} \Omega_{r}+\tau_{\phi} \\
I_{y y} \ddot{\theta}=\dot{\phi} \dot{\psi}\left(I_{z z}-I_{x x}\right)+J_{r} \dot{\phi} \Omega_{r}+\tau_{\theta} \\
I_{z z} \ddot{\psi}=\dot{\theta} \dot{\phi}\left(I_{x x}-I_{y y}\right)+J_{r} \Omega_{r}+\tau_{\psi} \\
m \ddot{x}=\left(S_{\psi} S_{\phi}+C_{\psi} S_{\theta} C_{\phi}\right) * u_{f}-\sum_{i=1}^{8} H_{x_{i}}-\frac{1}{2} C_{x} A_{c} \rho \dot{x} \mid \dot{x} \\
m \ddot{y}=\left(-C_{\psi} S_{\phi}+S_{\psi} S_{\theta} C_{\phi}\right) * u_{f}-\sum_{i=1}^{8} H_{y_{i}}-\frac{1}{2} C_{y} A_{c} \rho \dot{y} \\
m \ddot{z}=C_{\theta} C_{\phi} * u_{f}-m g
\end{array}\right.
$$

\section{B. Control Strategy}

A control strategy for stabilizing the octorotor while hovering is presented in this section. The altitude and the yaw positions are controlled by a PID controller that makes use of observations obtained respectively from an ultrasonic sensor and an Inertial Measurement Unit (IMU):

$$
u=K_{p} e+K_{d} \dot{e}+K_{I} \int_{0}^{t} e(\tau) d \tau
$$

$K_{p}, K_{d}$ and $K_{I}$ are the controller's gains, and $e$ is the state error compared to the desired position.

The roll and pitch angles are controlled using saturation functions, where each state is bounded separately (9). The stability of this control law is proved in [18].

$$
\begin{gathered}
\tau_{\phi}=\frac{I_{x x}}{g}\left[\sigma_{p y}\left(k_{p y}\left(y-y_{d}\right)\right)+\sigma_{d y}\left(k_{d y} \dot{y}\right)-\right. \\
\left.\sigma_{p \phi}\left(k_{p \phi} \phi\right)-\sigma_{d \phi}\left(k_{d \phi} \dot{\phi}\right)\right] \\
\tau_{\theta}=-\frac{I_{y y}}{g}\left[\sigma_{p x}\left(k_{p x}\left(x-x_{d}\right)\right)+\sigma_{d x}\left(k_{d x} \dot{x}\right)-\right. \\
\left.\sigma_{p \theta}\left(k_{p \theta} \theta\right)-\sigma_{d \theta}\left(k_{d \theta} \dot{\theta}\right)\right]
\end{gathered}
$$

where $k_{p y}, k_{d y}, k_{p \phi}, k_{d \phi}, k_{p x}, k_{d x}, k_{p \theta}$ and $k_{d \theta}$ are positive gains, and $\sigma_{p y}, \sigma_{d y}, \sigma_{p \phi}, \sigma_{d \phi}, \sigma_{p x}, \sigma_{d x}, \sigma_{p \theta}$, and $\sigma_{d \theta}$ are saturation functions defined as follows:

$$
\left\{\begin{array}{lll}
\sigma_{b_{i}}(s)=b_{i} & \text { if } \quad s>b_{i} \\
\sigma_{b_{i}}(s)=s & \text { if } \quad-b_{i}<s<b_{i} \\
\sigma_{b_{i}}(s)=-b_{i} & \text { if } \quad s<-b_{i}
\end{array}\right.
$$

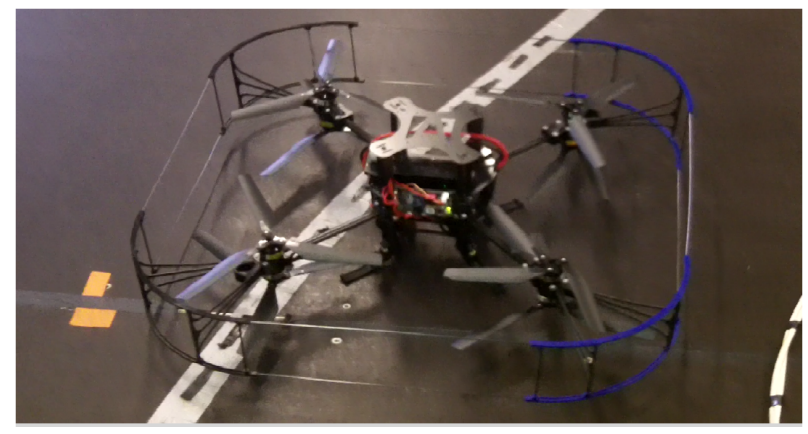

Fig. 2: Experimental Plateform

\section{MODEL'S PARAMETERS IDENTIFICATION}

The main objective of this section is to assess the applicability of the model developed previously to the coaxial counter-rotating octorotor. The parameters of the system are identified and validated using the mean square error criterion using real flight data sets. The following part describes our experimental octorotor, then the three subsystems used to model the thrust and translational dynamics. Furthermore, an overview of the used method, data and tests is given.

\section{A. Experimental Octorotor}

The experimental UAV is shown in Fig. 2. It is a coaxial $\dot{y}$ ctorotor built at the Heudiasyc laboratory. It uses $B l 2827-$ 35 brushless motors driven with $B L C T R L V 2$ controllers (Mikrokopter) giving motors speeds measurements.

The multirotor is equiped with a Microstrain $3 D M G X 3-$ 25 IMU composed of accelerometer, gyroscope, and magnetometer sensors giving Euler angles and rotation speed measurements at $100 \mathrm{~Hz}$, and an ultrasonic sensor $S R F 08$ giving altitude measurements. The control law is executed in real time onboard the vehicle.

The octorotor's inertia was extracted from the software Catia and was found to be as follows: $I_{x x}=I_{y y}=$ $4.2 \times 10^{-2} \mathrm{Kg} \cdot \mathrm{m}^{2}, I_{z z}=7.5 \times 10^{-2} \mathrm{Kg} \cdot \mathrm{m}^{2}$. The propeller's inertia was neglected. The vehicle's mass was measured to be $1.6 \mathrm{~kg}$, and the distance from the center of mass to the center of the propellers is $l=0.23 \mathrm{~m}$.

\section{B. Methodology}

Depending on the types of available data, and the way they are adopted, different approaches can be used for parameters identification [15]. The Trial-and-Error method is one of these approaches that has been widely used for model calibration and system identification. This technique is presented in Fig. 3 and is based on two main criterias:

C-1 The model output should fit the observed output as close as possible

C-2 The prior information should be used as much as possible

To use this method, only some observation data of the state variables with prior information of the identified parameters are needed. 


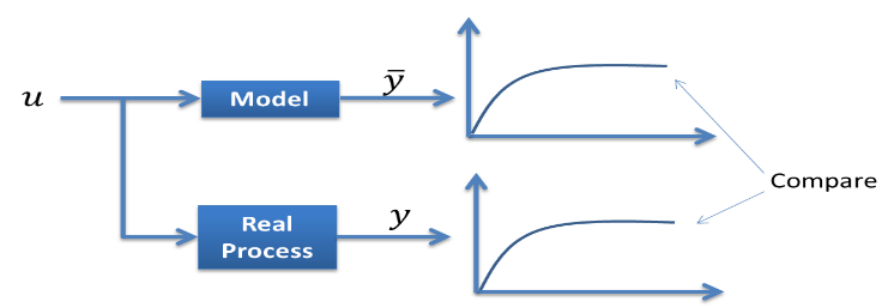

Fig. 3: Trial and Error Method

To guarantee the first criteria, we chose to minimize the mean square error $M S E$ function:

$$
M S E=\frac{1}{n} \sum_{i=1}^{n} e_{i}^{2}
$$

where $e_{i}=\hat{Y}_{i}-Y_{i}$ denotes the error between the estimated and the measured values at sample $i . Y_{i}$ corresponds in this case to the variables $x, y, z$ and their derivatives.

The procedure starts with a set of estimated values of all the parameters, which will be used as inputs to the forward model to generate the model-calculated system state. Then these calculated states will be compared to the observed data. New parameters that minimize the mean square error will be used instead of the initial guesses.

\section{Experimental Data}

To collect the needed data, different experiments were realized on the octorotor while it was moving along single directions or following complex trajectories. For each axis, one experiment is used for identification and another one for validation. Then, the complete model is validated on a complex trajectory. The measured variables used are:

1) Speeds of the eight motors at each instant;

2) Variations of roll, pitch and yaw angles $\phi, \theta$ and $\psi$;

3) Variations of translational velocities $\dot{x}, \dot{y}$ and $\dot{z}$;

4) Variations of translational displacements $x, y$ and $z$;

\section{Results and Model Validation}

1) Modeling along z-axis: The differential equation governing the motion of the vehicle along the $z$-axis is given in (12):

$$
m \ddot{z}=C_{\theta} C_{\phi} * u_{f}-m g
$$

The control input $u_{f}$ is the total thrust. It depends on the motors speeds and the thrust coefficient $K_{f}$.

In most of the works presented in the literature, the thrust coefficient $K_{f}$ is considered constant. However, this is just an hypothesis to simplify the model. It was shown from the experimental data collected that the thrust coefficient depends slightly on the motors speeds.

Figure 4 shows the variation of the thrust coefficient in terms of the motors speeds. The model parameters obtained from the experiments shown in Fig. 6 are applied to a second experiment, as shown in Fig. 7. In this experiment, the UAV is driven with respect to $z$-axis from $z=0$ to $z=0.7 \mathrm{~m}$.

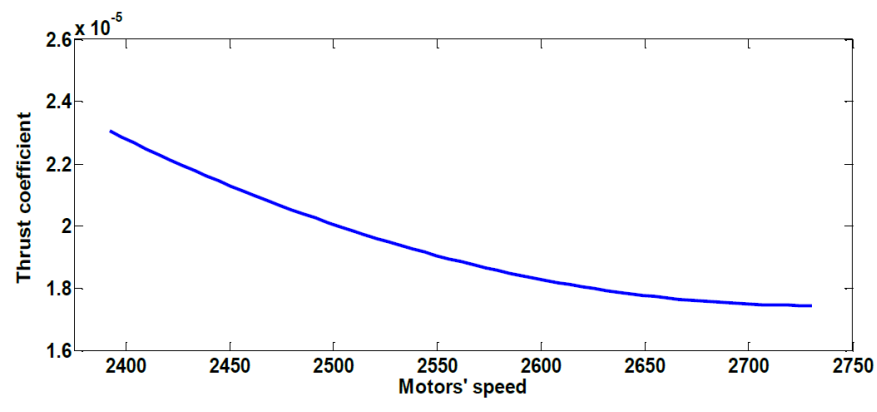

Fig. 4: The variation of the thrust coefficient $K_{f}\left[N s^{2} / \mathrm{rad}^{2}\right]$ in function of the motors speeds [rpm]

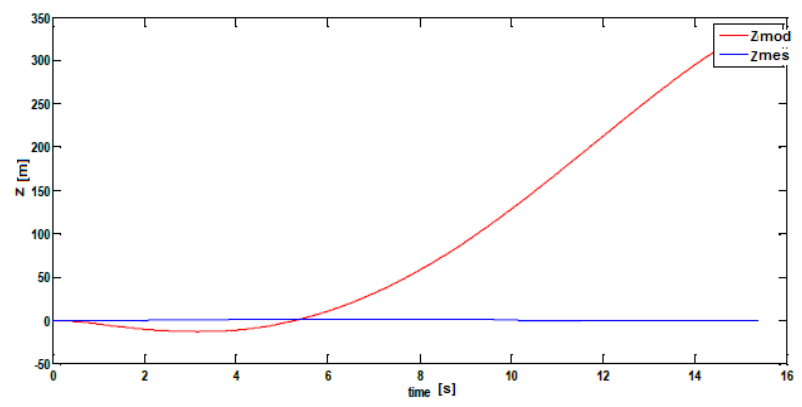

Fig. 5: The measured and the model-calculated altitude using the simplified model $[\mathrm{m}]$

Note that the sensor is placed at an altitude of $10 \mathrm{~cm}$ far from the ground.

The results in Fig. 7 show a significant error between the measured and the estimated altitude. However, their comparison with respect to the results obtained from the simplified model in Fig. 5 make the estimated model more representative of the real one. The causes of the shown error are discussed later in the paper.

2) Modeling along $x$-axis: For the model identification along the $x$-axis, the octorotor is considered to move along the $x$-direction at a constant altitude. The motors speeds vary slowly but they remain within the same rank, then the thrust coefficient is taken as a constant, $K_{f}=1.7 * 10^{-} 5$, deduced from the dataset presented above. The equation governing

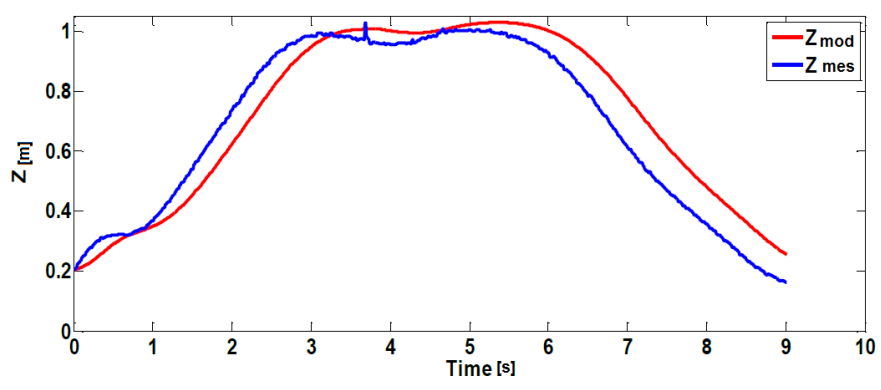

Fig. 6: Experiment 1 along $z$-axis: The measured and the model-calculated altitude $[\mathrm{m}]$ 


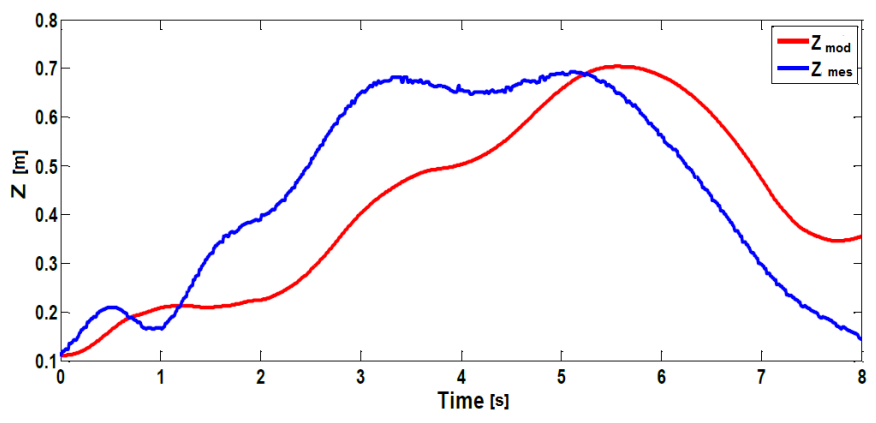

Fig. 7: Experiment 2 along $z$-axis: The measured and the model-calculated altitude [m]

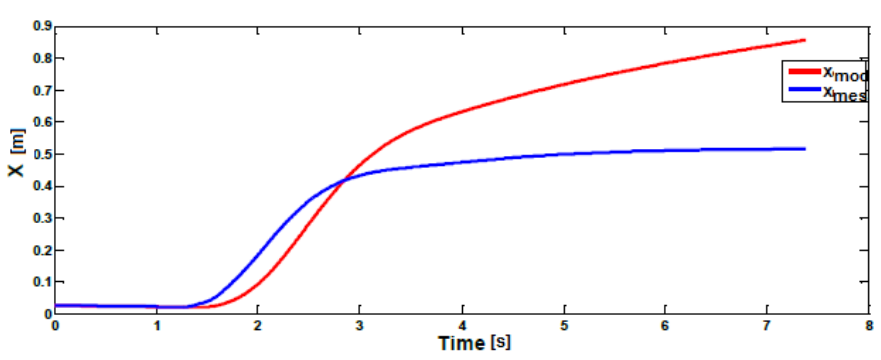

Fig. 8: The measured and the model-calculated position using the simplified model $\left[\mathrm{m} / \mathrm{s}^{2}\right]$

the motion along the $x$-axis is presented as follows:

$$
m \ddot{x}=\left(S_{\psi} S_{\phi}+C_{\psi} S_{\theta} C_{\phi}\right) * u_{f}-\sum_{i=1}^{8} H_{x_{i}}-\frac{1}{2} C_{x} A_{c} \rho \dot{x}|\dot{x}|
$$

The identified parameters are:

$$
\begin{aligned}
\sum_{i=1}^{8} H_{x_{i}} & =C_{H} \rho A\left(\Omega R_{\text {rad }}\right)^{2}=10^{-9} \cdot \sum_{i=1}^{8} \omega_{i}^{2} \\
\frac{1}{2} C_{x} A_{c} \rho x|\dot{x}| & =0.4688 \dot{x}|\dot{x}|
\end{aligned}
$$

First, Fig. 8 reveals the invalidity of the simplified model by comparing its output along $x$-axis with the measured one. Figures 9 and 10 show the model-calculated and the measured accelerations and positions. This experiment was used for the identification. For the validation purpose, data of a second experiment were used and the results are shown in Fig. 11 and 12.

3) Modeling along y-axis: The same procedure as the modeling along the $x$-axis is followed here. Figures 13 and 14 represent the data of the experiment used for identification, and Fig. 15 shows the validated model output compared with the measured state on a different experiment. The identified model parameters are approximately the same as those determined in (14). This is due to the system symmetry.

4) Validation over Complex Motion: To validate the identified model, the octorotor had to follow a complex trajectory with a movement along the three axes at the same time. The identified translational states $x, y$ and $z$ are shown respectively in Fig. 16, 17 and 18 and compared with the real measurements. Figure 19 shows the estimated and measured

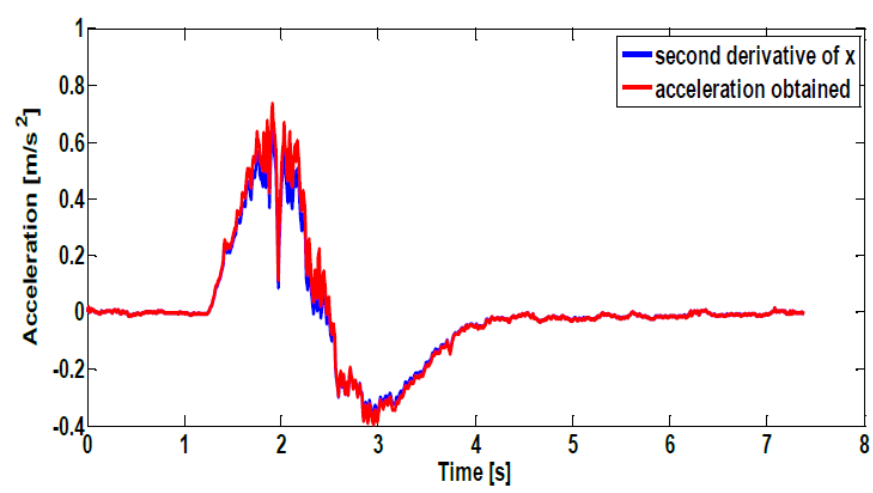

Fig. 9: Experiment 1 along $x$-axis: The measured and the model-calculated acceleration $\left[\mathrm{m} / \mathrm{s}^{2}\right]$

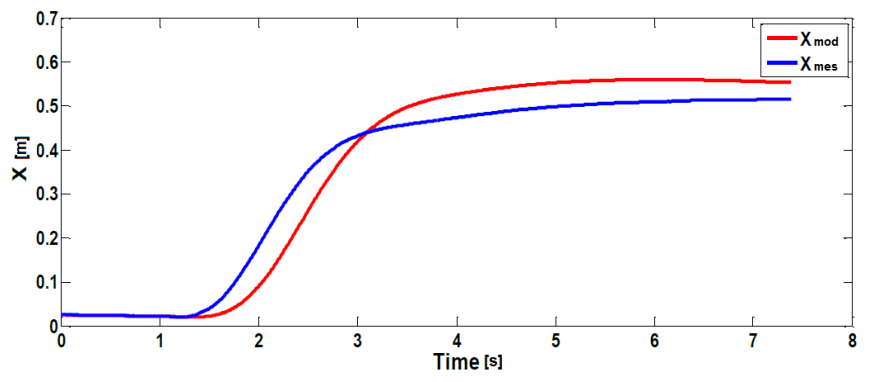

Fig. 10: Experiment 1 along $x$-axis: The measured and the model-calculated position $[\mathrm{m}]$

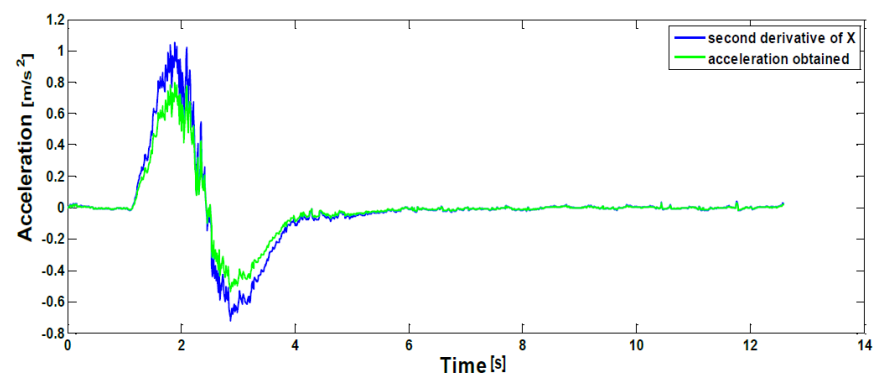

Fig. 11: Experiment 2 along $x$-axis: The measured and the model-calculated acceleration $\left[\mathrm{m} / \mathrm{s}^{2}\right]$

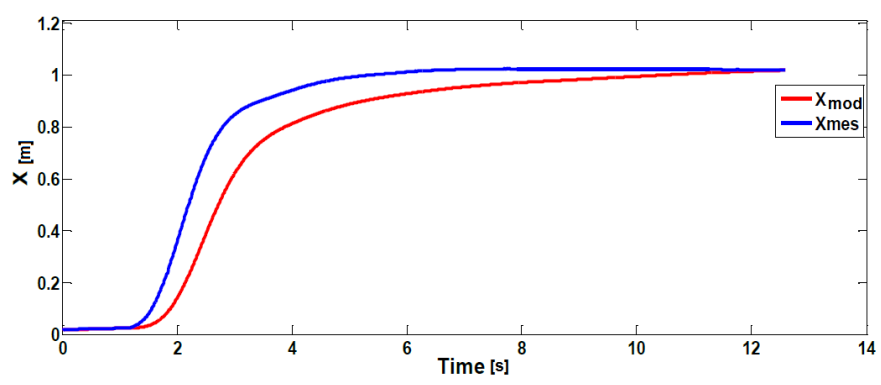

Fig. 12: Experiment 2 along $x$-axis: The measured and the model-calculated position $[\mathrm{m}]$ 


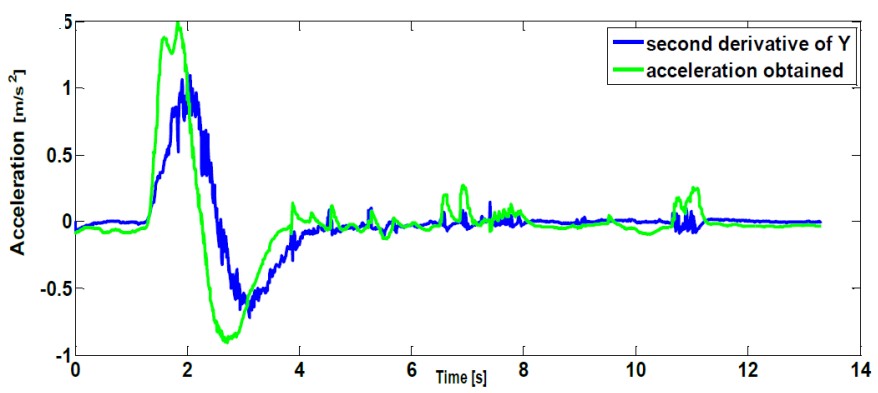

Fig. 13: Experiment 1 along $y$-axis: The measured and the model-calculated acceleration $\left[\mathrm{m} / \mathrm{s}^{2}\right]$

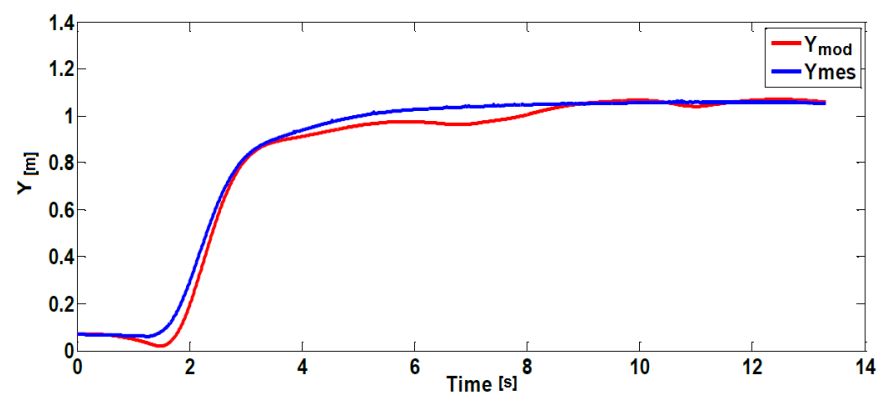

Fig. 14: Experiment 1 along $y$-axis: The measured and the model-calculated position $[m]$

trajectories on the three axes.

As can be seen in the plots of the validation, the identified dynamical model approximates well the behavior of the real plant but does not match it. This is caused by the fact that the identification errors are amplified by the double integration, add to this the delay introduced by the integrator in matlab.

\section{Discussion}

A well identified dynamic model of a multirotor UAV helps researchers to design controllers and predict the behaviors of the UAV via simulation. Although a best solution cannot be obtained by the trial-and-error method, an approximate model close to the real one can be deduced. This model can be sufficient for designing controller and especially for designing mathematical observers.

For many applications, such as fault detection and isolation, the use of a complete analytical model, including aerodynamical and gyroscopical effects, is necessary in

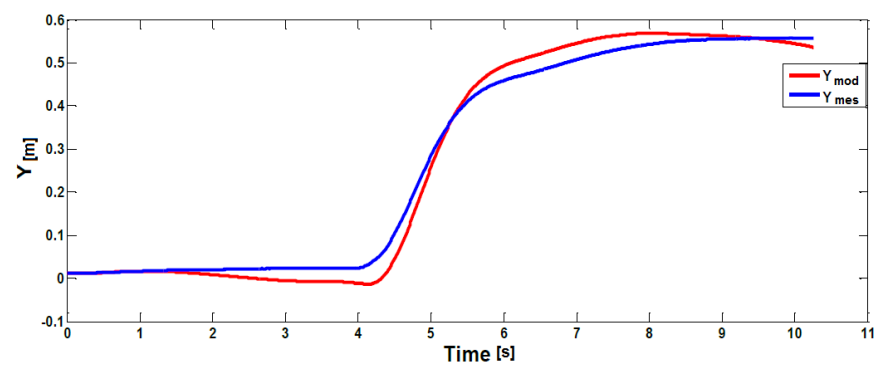

Fig. 15: Experiment 2 along $y$-axis: The measured and the model-calculated position $[\mathrm{m}]$

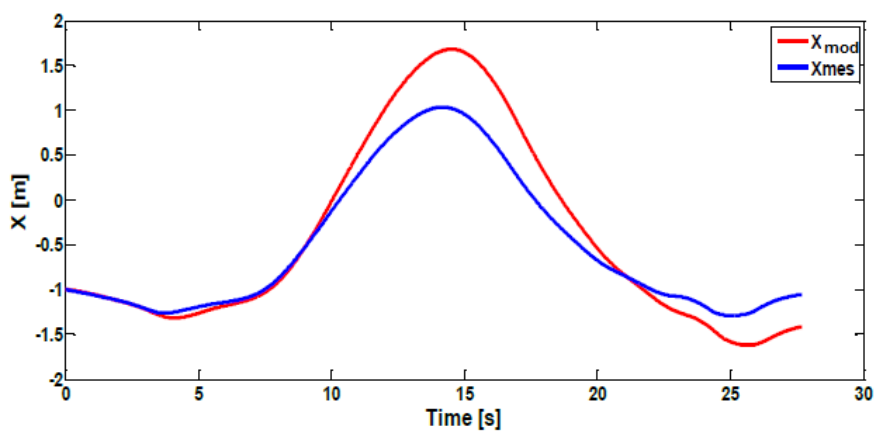

Fig. 16: Experiment along a complex motion: The measured and the model-calculated positions $[\mathrm{m}]$

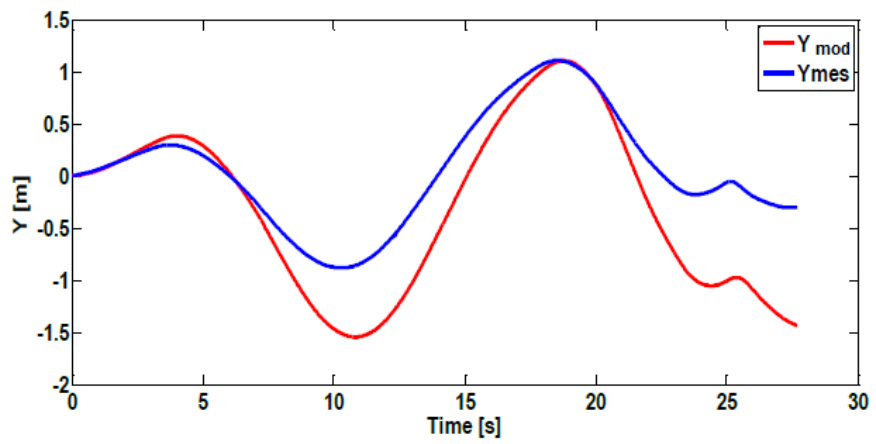

Fig. 17: Experiment along a complex motion: The measured and the model-calculated positions $[\mathrm{m}]$

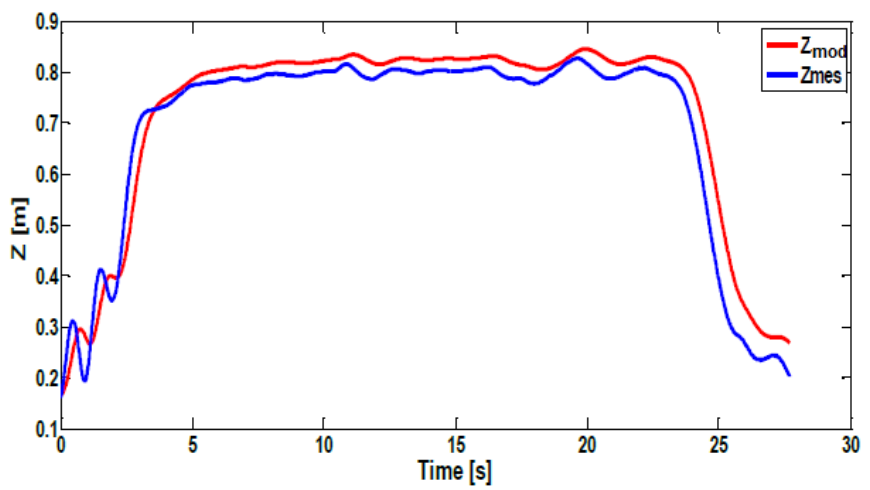

Fig. 18: Experiment along a complex motion: The measured and the model-calculated positions $[\mathrm{m}]$

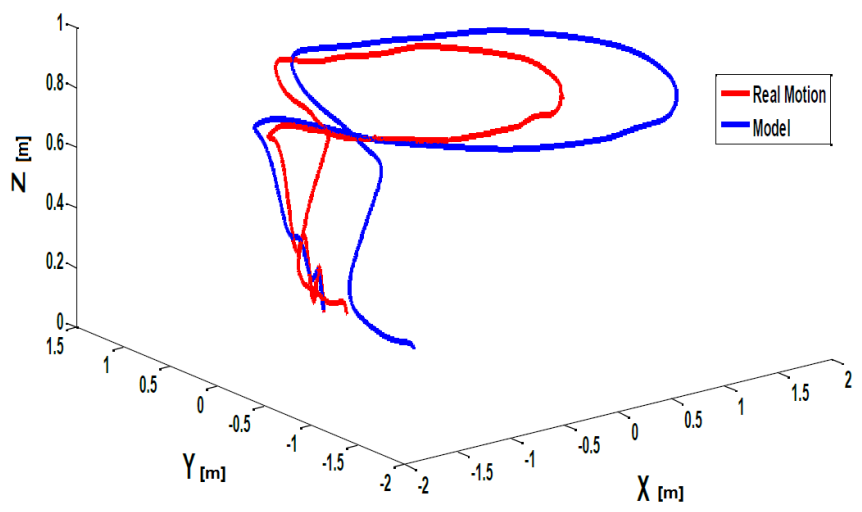

Fig. 19: Experiment along a complex motion: The measured and the model-calculated positions $[\mathrm{m}]$ 
order to design observers for fault estimation or residual generation.

In a previous work [16], we have proposed the use of a second-order sliding mode observer based on the SuperTwisting algrithm for fault diagnosis purposes in a octorotor UAV after the occurrence of motors failures. However, we could only validate it during hovering flights, as we used a simpler observer model that neglected aerodynamical effects. When the octorotor is moving, a more precise model is needed, such as the one presented in this paper.

\section{CONCLUSiON}

In this paper, a complete translational dynamic model of a coaxial counter-rotating octorotor UAV is proposed for special cases which are under the assumptions of small displacements without agressive maneuvers. By minimizing the mean square errors between the modeled states and the real ones, by trial-and-error method, the model parameters are identified. This makes possible the use of the obtained model for analytical observers designed to detect and isolate abnormal behavior or failure in the system.

In future works, we intend to complete the identification of the rotational model, by investigating other identification methods and considering different data sets. Finally, the obtained model will be used to design observers to estimate faults and failures in the system.

\section{ACKNOWLEDGEMENTS}

This work was carried out and funded in the framework of the Labex MS2T (Reference ANR-11-IDEX-0004-02) and the ROBOTEX Equipment of Excellence (Reference ANR-10- EQPX-44). They were supported by the French Government, through the program Investments for the future managed by the National Agency for Research.

The authors would like to thank the Lebanese University research support program and the Lebanese Council for Scientific Reasearch (LCSR).

\section{REFERENCES}

[1] S. Bouabdallah, "Design and control of quadrotors with applications to autonomous flying," $\mathrm{PhD}$ thesis, Ecole Polytechnique Fédérale De Lausanne, 2007.

[2] T. Schneider et al., "Fault-tolerant Control Allocation for Multirotor Helicopters Using Parametric Programming," International Micro Air Vehicle Conference and Flight Competition, Braunschweig, Germany, July, 2012.

[3] H. Alwi and C. Edwards, "Fault Tolerant Control of an Octorotor Using LPV based Sliding Mode Control Allocation," American Control Conference (ACC), Washington, DC, USA, June 17-19, 2013, pp. 65056510.

[4] A G Sidea, R Yding Brogaard, N A Andersen and O Ravn, "General model and control of an $n$ rotor helicopter," Journal of Physics: Conference Series 570, doi:10.1088/1742-6596/570/5/052004.

[5] F. Rinaldi, "Automatic control of a multirotor," PhD Thesis, POLITECNICO DI TORINO, Mars 2014.

[6] A. Freddi, A. Lanzon, and S. Longhi, "A feedback linearization approach to fault tolerance in quadrotor vehicles," IFAC World Congress, Milano, Italy, 2011, pp. 5413-5418.

[7] T. Madani, A. Benallegue, "Backstepping Control for a Quadrotor Helicopter," IEEE/RSJ International Conference on Intelligent Robots and Systems, Beijing, Oct. 9-15, 2006, pp. 3255-3260.
[8] P. Castillo, A. Dzul and R. Lozano, "Real-time stabilization and tracking of a four rotor mini rotorcraft," IEEE Transactions on Control Systems Technology, vol. 12, issue 4, pp. 510-516, 2004.

[9] J. Escareno, S. Salazar-Cruz, and R. Lozano, "Embedded control of a four-rotor uav," American Control Conference ACC, Minneapolis, MN, 14-16 June 2006, pp. 3936-3941.

[10] M. Oliveira, "Modeling, Identification and Control of a Quadrotor Aircraft," Master Thesis, Czech Technical University in Prague, 2011.

[11] T. Bresciani, "Modelling, Identification and Control of a Quadrotor Helicopter," Master Thesis, Lund University, 2008.

[12] D. Miller, "Open Loop System Identification Of a Micro Quadrotor Helicopter From Closed Loop Data," Master Thesis, University of Maryland, 2011.

[13] P. Castillo, R. Lozano and A. Dzul, "Modelling and control of miniflying machines," Springer-Verlag New York Inc, 2005.

[14] F. Kendoul, D. Lara, I. Fantoni and R. Lozano, "Nonlinear control for systems with bounded inputs: Real-time embedded control applied to UAVs," IEEE Conference on Decision and Control CDC, San Diego, CA, USA, December 13-15, 2006, pp. 5888-5893.

[15] N.-Z. Sun and A. Sun, "Model Calibration and Parameter Estimation For Environmental and Water Resource Systems," Springer-Verlag New York, 2015

[16] M. Saied, H. Shraim, C. Francis and B. Lussier, "Actuator Fault Diagnosis in an Octorotor UAV Using Sliding Modes Technique: Theory and Experimentation," Europeen Control Conference ECC, Linz, Austria, July 2015

[17] A. Koehl, "Modélisation, Observation et Commande d'un drone miniature birotor coaxial," Ph.D thesis, University of Nancy, 2012.

[18] G. Sanahuja, P. Castillo and A. Sanchez, "Stabilization of $\mathrm{n}$ integrators in cascade with bounded input with experimental application to a VTOL laboratory system," Int. Journal of Robust and Nonlinear Control, vol. 20, no. 10, pp. 1129-1139, 2010. 